\title{
Fine-scale genetic mapping of a hybrid sterility factor between Drosophila simulans and D. mauritiana: the varied and elusive functions of "speciation genes"
}

\author{
Luciana O Araripe*, Horácio Montenegro, Bernardo Lemos, Daniel L Hartl
}

\begin{abstract}
Background: Hybrid male sterility (HMS) is a usual outcome of hybridization between closely related animal species. It arises because interactions between alleles that are functional within one species may be disrupted in hybrids. The identification of genes leading to hybrid sterility is of great interest for understanding the evolutionary process of speciation. In the current work we used marked P-element insertions as dominant markers to efficiently locate one genetic factor causing a severe reduction in fertility in hybrid males of Drosophila simulans and D. mauritiana.

Results: Our mapping effort identified a region of $9 \mathrm{~kb}$ on chromosome 3, containing three complete and one partial coding sequences. Within this region, two annotated genes are suggested as candidates for the HMS factor, based on the comparative molecular characterization and public-source information. Gene Taf1 is partially contained in the region, but yet shows high polymorphism with four fixed non-synonymous substitutions between the two species. Its molecular functions involve sequence-specific DNA binding and transcription factor activity. Gene agt is a small, intronless gene, whose molecular function is annotated as methylated-DNA-protein-cysteine S-methyltransferase activity. High polymorphism and one fixed non-synonymous substitution suggest this is a fast evolving gene. The gene trees of both genes perfectly separate $D$. simulans and $D$. mauritiana into monophyletic groups. Analysis of gene expression using microarray revealed trends that were similar to those previously found in comparisons between whole-genome hybrids and parental species.
\end{abstract}

Conclusions: The identification following confirmation of the HMS candidate gene will add another case study leading to understanding the evolutionary process of hybrid incompatibility.

\section{Background}

Reproductive isolation is a hallmark of speciation in sexual organisms. When genetically isolated populations have accumulated enough divergence, the hybrid progeny may be sterile due to the disruption of gametogenesis caused by functional incompatibility between factors evolved independently within each population. This scenario characterizes post-zygotic isolation, which is frequently found in pairs of species sharing a recent common ancestor. However, the timing at which

\footnotetext{
* Correspondence: lararipe@fas.harvard.edu
Department of Organismic and Evolutionary Biology, Harvard University,

* Correspondence: lararipe@fas.harvard.edu
Department of Organismic and Evolutionary Biology, Harvard University, Cambridge, Massachusetts 02138, USA
}

(c) 2010 Araripe et al; licensee BioMed Central Ltd. This is an Open Access article distributed under the terms of the Creative Commons Attribution License (http://creativecommons.org/licenses/by/2.0), which permits unrestricted use, distribution, and reproduction in any medium, provided the original work is properly cited. ciation is somewhat unclear.

An evolutionary scenario of speciation was theorized many decades ago [1,2], but a modern understanding of the speciation process on the molecular level-identifying the so-called "speciation genes"-has just begun to become realistic. A number of studies have succeeded in identifying, at the molecular level, a few genes that may be involved in speciation (in Drosophila: OdsH $[3,4]$, Nup98 [5], Nup160 [6], Hmr [7], Zhr [8], Ovd [9]; in Mus: Prdm9 [10], in Xiphophorus: Xmrk-2 [11]). These recent data together provide needed insight into the evolution of reproductive isolation, and they largely confirm the traditional view of speciation as an evolutionary 
process involving multiple genes [12,13]. Moreover, the results imply that speciation is a continuous process that progresses from the occurrence of hybridization with viable hybrids, to hybrid sterility, and ultimately to complete pre-zygotic reproductive isolation. Thus, the existence of multiple reproductive barriers that have accumulated over time is expected [12].

According to the Dobzhansky-Muller model $[1,2]$, genetic incompatibilities arise from negative epistatic interactions between alleles that have appeared within each population and encountered each other for the first time in the hybrid. Thus, sequence differences within at least two loci between two closely related species is a prerequisite for genetic incompatibility. Indeed, the bigger the differences in gene sequence between species, the higher the likelihood that an incompatible sequence variant may have arisen. In this sense, every gene showing rapid evolution might potentially be responsible for generating the incompatibilities in the hybrid of two closely related species.

The abundance of complex epistatic interactions involved in HMS has been recently shown [14-16]. For instance, previous work on the same $D$. simulans $/ D$. mauritiana system used here uncovered several complex epistatic interactions between HMS factors, even though the analysis was restricted to small introgressions in a single background. This limited the results to interactions between factors located not too distant from each other [16]. Nevertheless, the number of genes usually involved and the nature of the epistatic interactions are yet to be resolved.

Although hybrid inviability and/or sterility are the usual outcomes of the disruption of allelic interactions, the sparse data so far accumulated indicate that the underlying nature of the disruptions may vary. The question of whether certain classes of genes are more prone to evolve incompatibilities is still open. Further studies are therefore likely to bring new insights to the topic of speciation. The number and variety of genomic regions found to be involved in some degree of hybrid incompatibility suggest that most of the divergence between species may have accumulated after the rise of reproductive barriers $[13,17,18]$, and indeed several studies have observed an increase in the number of incompatibilities with divergence time $[19,20]$.

Genes causing hybrid inviability may have important housekeeping, developmental, or regulatory functions, whereas genes leading to sterility in hybrids would likely be involved in some aspect of reproduction. Among the genes described so far, three are DNA or chromatinbinding proteins (OdsH, $\mathrm{Lhr}$ and $\mathrm{Hmr}$ ), two are nuclear pore proteins (Nup96, Nup160), one is a gene transposition (JYalpha), and one is likely to be a small regulatory RNA that suppresses sex-ratio distortion (Nmy). As pointed out by Presgraves [21], genomes are not impervious to invasion by selfish elements, and substitutions generated by these leave the same signatures in the genome as beneficial substitutions. Therefore, an alternative to the hypothesis of adaptive evolution is that most of these genes may have evolved as a compensatory response to the effects of deleterious mutations and selfish genes.

In the current work we focus on locating one hybridmale-sterility (HMS) factor between $D$. simulans and $D$. mauritiana and investigating the nature of the disruption behind it. The HMS factor 1 was previously identified by Tao et al. [16] as being in a region of $1.4 \mathrm{Mb}$ on chromosome 3 (between molecular markers Rga and Antp). This is only one of ten factors in chromosome 3 possibly causing hybrid incompatibilities in this pair of species, whose hybrid males are always sterile and females are fertile. Our results show that a fertility shift from quasi-sterile male to a fertile male is associated with a region of $9 \mathrm{~kb}$, in which three complete genes and a portion of one gene are contained. We analyzed the DNA sequence across this interval and found no duplication, deletion, or rearrangement between the two species. However, we observed a handful of divergent sites in the coding sequences of gene CG17603 (Taf1) and the intronless 576 bp gene CG1303 (agt), as well as indels present in the 5' UTR of the later and in the intergenic region immediately upstream of its coding sequence. Gene agt shows a higher number of non-synonymous (NSS) than synonymous (SS) substitutions, one of the NSS being fixed between $D$. simulans and D. mauritiana; the gene also shows a reciprocally monophyletic gene tree. Likewise, the gene tree of Taf1, built from the portion of coding sequence included in the mapped interval, unambiguously separates the two species. The other two genes in the region are much more evolutionary conserved and have gene trees that do not differentiate the two species.

\section{Results}

\section{Mapping of the HMS factor to a $\mathbf{9} \mathrm{kb}$ genomic region}

Crosses of $2 P$-cis females with simB-males generated 536 recombinant males. This number was reached after three runs of crosses, genotyping, and phenotyping. As shown in Figure 1 recombinant males are recognized from the eye color corresponding to P32-insert and each bears either the original introgression or one of smaller size that may have been generated by recombination in the mother. In principle, the sizes of the introgressions were not known, and all males were tested by crossing to $P 45.6$ (tester stock) and by scoring the fertility of their $2 P$ sons (which carry both $P$-elements and introgressions). 


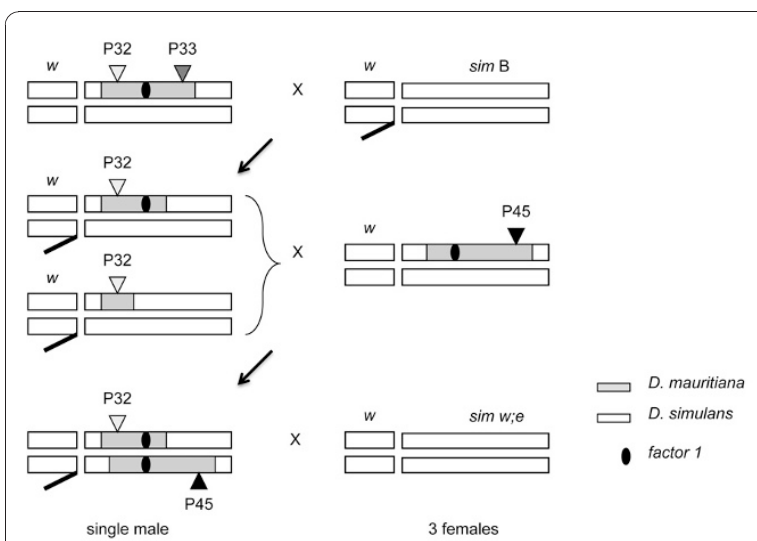

Figure 1 Mapping design and crosses performed. Cross scheme used to generate recombinants between two $P$-elements. The second chromosome, marked with $n t$ is not shown. Females from $2 P$ line 32-33 (see True et al. 1996a for P-element nomenclature) were crossed to simB males and recombinants were selected by eye color. Recombinants carrying P33 had eye color very similar to nonrecombinants, which made the selection difficult. Thus, we decided to select only recombinants carrying $P 32$ for the fertility tests. The D. mauritiana introgression did not cause sterility when heterozygous; so the recombinant lines were crossed to $1 P$ line 45.6 whose introgression covers the region where the location of factor 1 was predicted. Ten males carrying P32 and P45.6 from each cross, i.e. homozygotes for the introgression, were selected by eye color and individually crossed to 3 females $w$; e. Fertility was assayed by counting the offspring from each cross up to the $20^{\text {th }}$ day.

The screening of the first set of recombinants reduced the region of factor 1 from $1.26 \mathrm{Mb}$ (between markers Rga and Antp) to $372 \mathrm{~kb}$ (between markers CG15179 and Antp) and to chromosomal location 84A1. In the second run, we first genotyped recombinants using the ASO markers on the edge of factor 1's region (CG15179 and $A n t p)$ in order to select for the informative lines and exclude the lines with break points outside of the $372 \mathrm{~kb}$ interval. This new effort reduced the region to $170 \mathrm{~kb}$ (between markers CG15179 and Dfd). Finally, the third run helped us locating factor 1 within an interval of $20 \mathrm{~kb}$, with four informative recombinant lines within it. The smallest interval between the fertile line P32.433 and the quasi-sterile line P32.456 is $9 \mathrm{~kb}$ (Figure 2 and Additional file 1).

The phenotype corresponding to each genotype class is shown in Figure 2. We summarize the difference in phenotype by showing the mean progeny number for fertile recombinants $(215.4 \pm 24.51)$ and quasi-sterile recombinants $(9.0 \pm 2.32)$. This represents a reduction of 24-fold in fertility when the $9 \mathrm{~kb}$ region of $D$. mauritiana is present in homozygous condition.

The $9 \mathrm{~kb}$ region contains three annotated genes: CG1307, CG2358 (Spase 18-21) and CG1303 (agt). A fragment of gene CG17603 (Taf1, 3' end representing $44 \%$ of the gene and $23 \%$ of the transcript) is also included in the region. Additional file 2 shows the genes in the mapped interval. Gene Taf1 has molecular functions described as: sequence-specific DNA binding, general RNA polymerase II transcription factor activity, histone serine kinase activity, protein kinase activity, transcription factor activity, and zinc ion binding. Gene CG1307 has molecular function described as aminoacyltRNA hydrolase activity. Spase 18-21 has molecular function described as serine-type peptidase activity. Finally, agt has molecular function described as methylated-DNA-protein-cysteine S-methyltransferase activity.

DNA sequencing of the $9 \mathrm{~kb}$ region for $D$. simulans and $D$. mauritiana revealed no large duplication, deletion, or chromosomal rearrangement between the species. Across the region we see an even distribution of SNPs and indels, with a clearly higher conservation observed within coding regions (see Additional file 3). Gene Taf1 has seven of 16 exons included in the region, CG1307 and CG2358 have three exons each, and agt (CG1303) has one single exon. Single-nucleotide differences between species are spread across the whole region, but are especially seen in introns and intergenic regions. Comparing the coding sequences of the genes, both genes Taf1 and agt show a high density of singlenucleotide differences, whereas no indels were found within exons. The highest divergence between $\operatorname{simB}$ and mau12 is seen at the upstream region of gene agt. Within a range of $500 \mathrm{bp}$ from the 5' UTR of gene agt, we see four small indels, with simB missing a total of 54 bp in relation to mau12 (see Additional file 2).

\section{Molecular characterization of the candidate interval}

A count of the number of substitutions occurring in exons of the four genes for $D$. simulans and D. mauritiana allowed an informative analysis of the candidate interval. The results are summarized in Table 1. Genes CG1307 and CG2358 can be immediately excluded as candidates for the HMS phenotype, as they show no significant variation between species. Gene CG1307 shows a total of nine polymorphic sites and one silent substitution fixed between species. We performed a McDonaldKreitman test, which takes into account a neutral null hypothesis to test whether the ratio of synonymous to non-synonymous substitutions that are fixed between species is consistent with that segregating within species. Fisher's exact test was not significant (one-tailed $P=$ 0.5). Gene CG2358 (Spase 18-21) is very conserved, showing silent polymorphism, but no fixed differences between species (Table 1). Thus, the McDonaldKreitman test could not be performed. Moreover, the gene trees for CG1307 and CG2358 do not separate the two species into monophyletic groups (Figure 3B,C).

Contrary to CG1307 and CG2358, the number of substitutions seen for gene Taf1 is noticeable. In the 


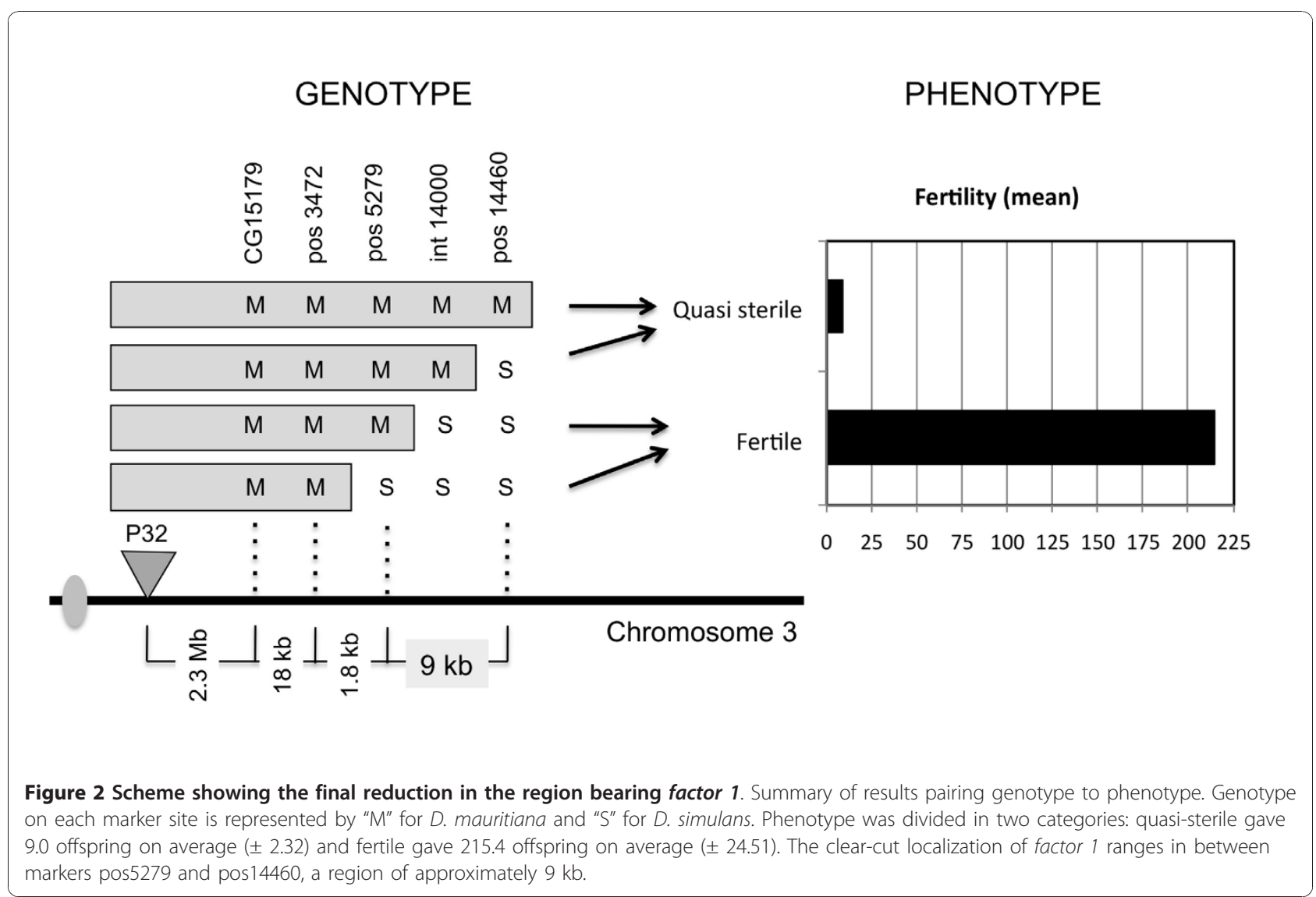

relatively small portion of Taf1's coding sequence included in the region ( $23 \%$ of $6.4 \mathrm{~kb}$ ), we find four non-synonymous substitutions that are fixed between species, in addition to the occurrence of 22 polymorphic sites (Table 1). The gene tree constructed for Taf1 unambiguously separates the species $D$. simulans and D. mauritiana (Figure 3A). Even though the sequence analysis and gene tree suggest that Taf1 may be under rapid evolution, and hence be a good candidate for the hybrid incompatibility, this is a gene that shows relatively high conservation across species of the D. melanogaster group (see Additional file 3). Moreover, from among 23 loss-of-function mutations found in a genetic screen of Taf1, three were identified as causing female sterility without affecting the fertility of males [22]. The other alleles cause lesions in a variety of structures, including bristles, wings and male terminalia, and some may be lethal.

The other candidate for factor 1, the gene agt, also shows a high density of single-nucleotide substitutions (Table 1), including 15 that are non-synonymous. Also, the 5' UTR and upstream intergenic region of agt show the highest divergence between species, mostly in the form of indels. Conservation in this particular region (D. simulans 3R: 2517094-2518594) is low across species of the melanogaster subgroup (see Additional file 3) and

Table 1 Molecular characterization of the candidate interval

\begin{tabular}{|c|c|c|c|c|c|c|}
\hline & Polymorphism within species & Fixed differences between species & McDonnald-Kreitman & $K a$ & Ks & $K a / K s$ \\
\hline \multirow[t]{2}{*}{ Taf1 } & $S S=22$ & $\mathrm{SS}=8$ & $P=0.5$ & 0.0039 & 0.034 & 0.114 \\
\hline & NSS $=10$ & $\mathrm{NSS}=4$ & & & & \\
\hline \multirow[t]{2}{*}{ CG1307 } & $\mathrm{SS}=5$ & $\mathrm{SS}=1$ & $P=0.5$ & 0.0039 & 0.020 & 0.198 \\
\hline & $\mathrm{NSS}=4$ & $\mathrm{NSS}=0$ & & & & \\
\hline \multirow[t]{2}{*}{ CG2358 } & $S S=7$ & $S S=0$ & NA & 0 & 0.012 & 0 \\
\hline & NSS $=0$ & NSS $=0$ & & & & \\
\hline \multirow[t]{2}{*}{ agt } & $S S=10$ & $\mathrm{SS}=4$ & $P=0.078$ & 0.009 & 0.047 & 0.194 \\
\hline & NSS $=15$ & $\mathrm{NSS}=1$ & & & & \\
\hline
\end{tabular}




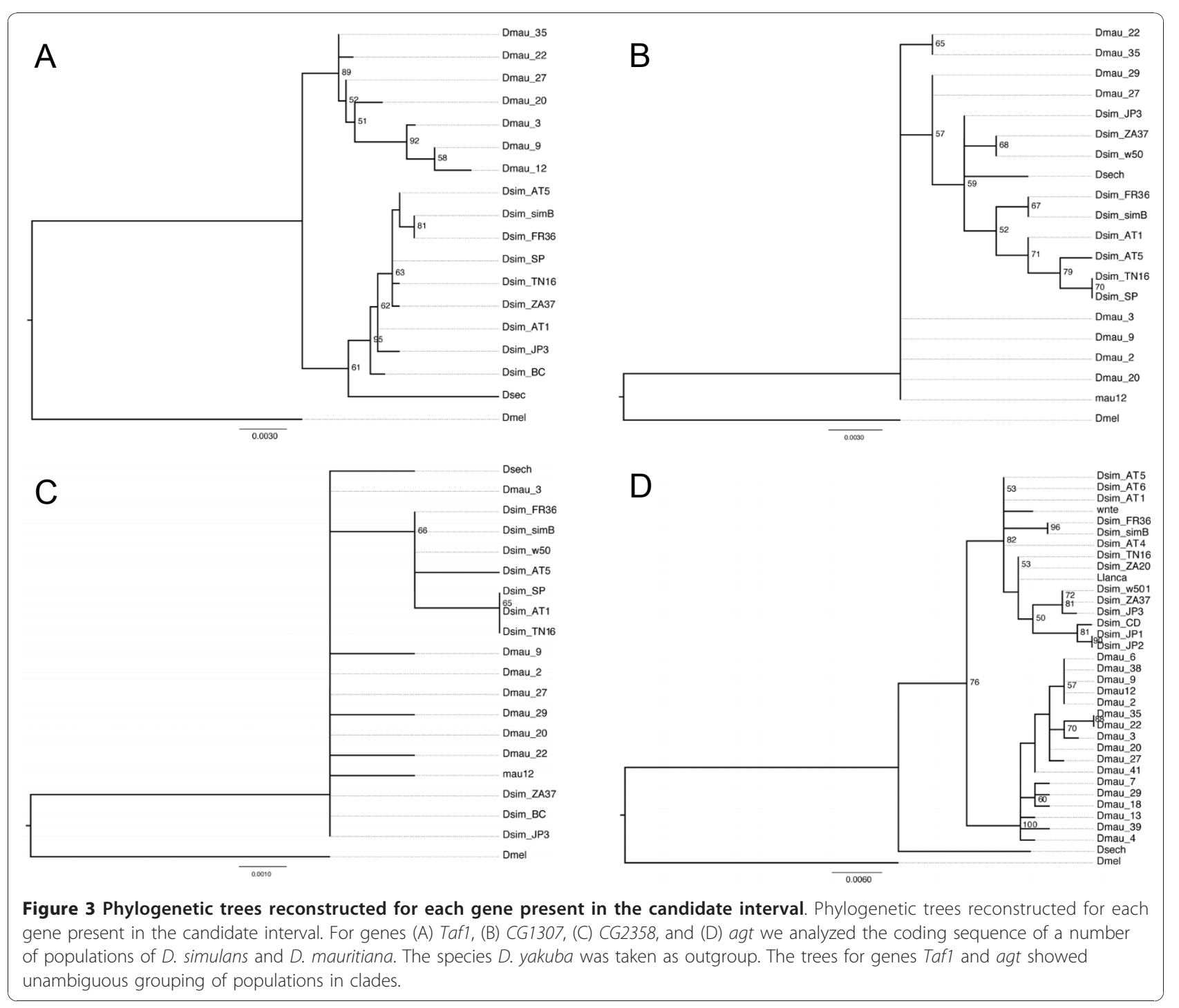

even between the sister species $D$. simulans and D. mauritiana.

Gene agt is only 576 bp long (191 amino acids) and has no introns. The protein O-6-alkylguanine-DNA alkyltransferase is involved in the repair of O-6-alkylguanine and O-4-alkylthymine in DNA, and in most organisms it attenuates the cytotoxic and mutagenic effects of certain classes of alkylating agents. Between D. simulans and $D$. mauritiana the coding region of gene agt shows a higher number of non-synonymous (NSS) than synonymous (SS) substitutions: D. simulans has 13 segregating sites with seven NSS, whereas D. mauritiana has 12 segregating sites with eight NSS. It is important to highlight that one non-synonymous substitution is fixed between species (Table 1). However, Fisher's exact test was not significant (one-tailed $P=0.078$ ).

In addition to its degree of sequence divergence, agt's gene tree is perfectly consistent with the separation of
D. simulans and D. mauritiana into monophyletic groups (Figure 3D), in contrast to the gene trees of CG1307 and CG2358. In fact, it is expected that genes involved in speciation will reflect more accurately the phylogenetic history of closely related species [23,24], and this indeed has been shown to be the case for another gene causing hybrid sterility, OdsH [25].

The non-silent difference between species at position 361 of agt's coding region is also a variable site when other pairs of species are considered (Table 2). D. erecta and D. yakuba have GAT (aspartic acid) in position 361, D. melanogaster has CAT (histidine), D. sechelia has TAT (tyrosine), D. mauritiana has AAT (asparagine), and $D$. simulans has GAT (aspartic acid) like the outgroup $D$. yakuba. This specific change may suggest a precise location for the origin of the hybrid incompatibility.

Our results point to genes Taf1 and agt as good candidates for the hybrid male incompatibility factor 1 
Table 2 Amino acid replacement in position 361 of the candidate gene agt

\begin{tabular}{ccc}
\hline Species & Codon at position $\mathbf{3 6 1}$ & Amino acid \\
\hline D. simulans & GAT & Aspartic acid (Asp) \\
D. mauritiana & AAT & Asparagine (Asn) \\
D. sechelia & TAT & Tyrosine (Tyr) \\
D. melanogaster & CAT & Histidine (His) \\
D. yakuba & GAT & Aspartic acid (Asp) \\
D. erecta & GAT & Aspartic acid (Asp) \\
\hline
\end{tabular}

mapped to the $9 \mathrm{~kb}$ region herein reported. However, the fact that gene Taf1 is not entirely represented in the mapped interval and, in D. melanogaster, affects the fertility of females and not males when disrupted, makes this gene a less attractive candidate for causing the male-sterile phenotype than it might otherwise be.

\section{Complex epistasis between HMS and the genomic background}

We have tested whether the HMS factor 1 was similarly expressed in three different genomic backgrounds. We find that significant variation in fertility is observed when other strains of $D$. simulans are used. In order to establish stocks for this experiment, males from recombinant lines $P 32.75$ and $P 32.110$, as well as males from the tester stock P45.6, were crossed to females from three different strains of $D$. simulans: w501, $w ; e$, and $y w f$. Male progeny with colored eyes were backcrossed to virgin females from the respective $D$. simulans strain for five more generations. Because no recombination happens in males of most species of Drosophila, at this point we expected that each original recombinant chromosome would be intact, whereas the rest of the genome would have been largely replaced by the background strain. Females from each background were then crossed to males from the corresponding $P 45.6$ line and the $2 P$ male progeny tested for fertility. The fertility tests were done as described in Material and Methods: each male $2 P$ was crossed to three females $w ; e$ and the progeny were counted. The results are shown in Figure 4. The $w ; e$ background gives a 30-fold difference in fertility between the fertile line P32.75 (factor 1 absent) and the quasi-sterile line P32.110 (factor 1 present in homozygosis). The smallest effect of factor 1 was seen in the w501 background (2-fold), even though the difference in progeny size is still highly significant $(P<0.01)$.

\section{Gene expression analysis}

Patterns of gene expression may also give clues as to the molecular nature of factor 1. For this, we began by investigating the tissue-specificity of the four genes contained within the introgressed region using publicly available data for D. melanogaster (FlyAtlas [26]). Gene

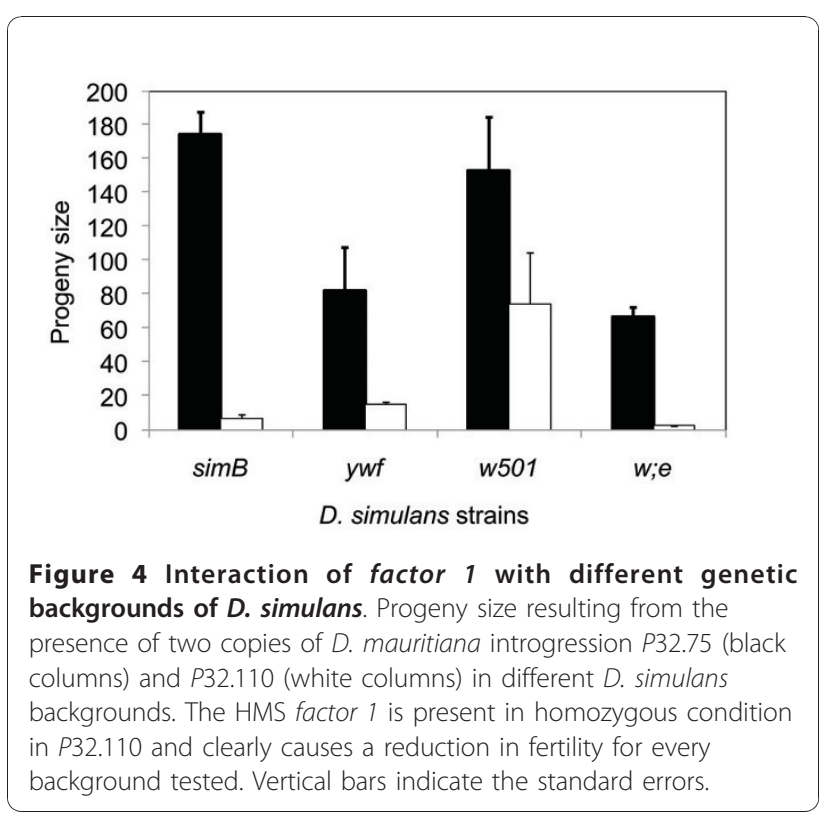

Taf1 is expressed at low and similar levels in all tissues; expression level in testes is basal and reported to be half that for ovaries. Gene CG1307 has a developmentally homogeneous expression pattern that appears to be restricted to tubule and hindgut tissues. Taken together with its lack of evolutionary variability, this pattern appears sufficient to rule it out as the cause of the HMS herein observed. Gene CG2358 is ubiquitously expressed although levels vary greatly across tissues. Its highest expression level is in salivary glands. Moreover, its high sequence conservation across species allows us to rule it out as a cause for HMS. Finally, gene agt is expressed at low levels and just above the detection limit in various tissues. Importantly, in the male accessory gland, agt is expressed in levels at least two times higher than most of the other tissues. Male accessory glands are required for sperm storage and male fertility.

Microarray analysis helped identifying the molecular correlates of the hybrid male sterility and showed a substantial number of gene expression differences associated with the non-fertile phenotype relative to the fertile phenotype (Figure 5). First, we found 932 genes whose expression varies between the fertile and nonfertile lines $(P<0.01, F D R<0.10$, see Additional file 4$)$. Furthermore, we found that 157 genes $(F D R<0.05)$ show concordant gene expression differences in all four non-fertile lines relative to the fertile line (see Additional file 5), with 124 genes similarly down-regulated in all four non-fertile lines and only 33 genes similarly upregulated in the same lines - see also Figure 5. Importantly, the genes affected are randomly scattered throughout the genome with only 5 down-regulated targets contained within the introgressed segment. 


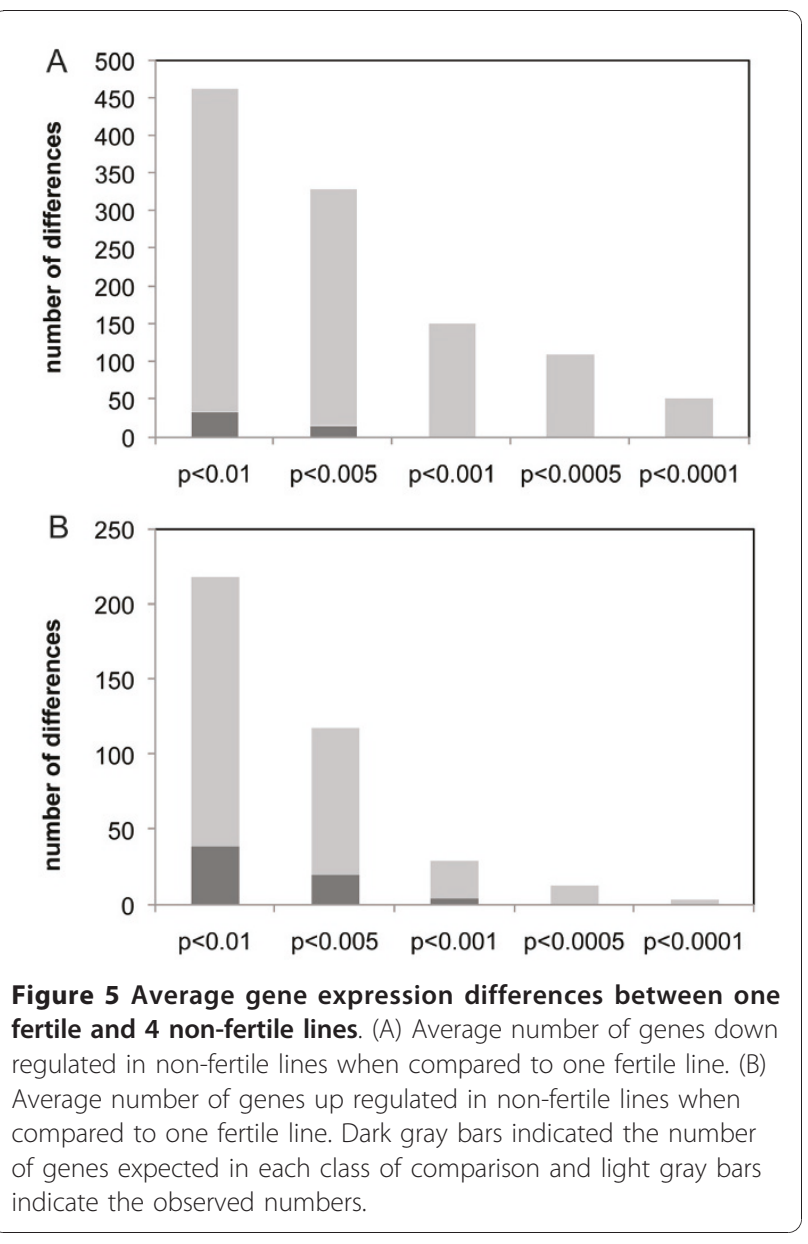

Altogether, the genes differentially expressed between lines underscore the regulatory networks that are disrupted by factor 1 or other divergent elements in the mapped region. Interestingly, while the genes detected show a broad distribution of functional classes, we detected a statistically significant enrichment for genes whose functions are associated with spermatogenesis ( $P=0.002$, Fisher's exact test). Accordingly, we observed 19 downregulated genes and 11 upregulated genes in non-fertile lines and belonging to the gene ontology category of "spermatogenesis".

\section{Discussion}

The history of divergence of $D$. simulans and $D$. mauritiana from a common ancestor dates from $\sim 0.3$ million years ago [27] and likely happened through the common mechanism of allopatric speciation [28]. The distribution of the two species overlapped recently, at some point around 24,000 years ago, as evidenced by an introgression of $D$. simulans mtDNA into D. mauritiana [29]. The rise of reproductive isolation in this system has been the object of several studies. Hybrid male sterility (HMS) loci have been found mainly on the X chromosome (reviewed in Wu \& Hollocher [30]), but Tao et al. [16] have described the occurrence on the $3^{\text {rd }}$ chromosome of 10 HMS factors by genetic mapping, among a total of 19 quantitative trait loci (QTL) in this chromosome that may be involved in hybrid incompatibilities.

The use of QTL mapping to identify the genomic region responsible for the expression of a complex phenotype has been extensive in several organisms. Because hybrid sterility as a complex trait may result from disruptions in varied genes and genetic interactions, the use of a mapping approach that mixes two genomes in equal proportions is very likely to give no fertile individuals. Nevertheless, the use of introgressions of one species in the genomic background of another species has been effective in the search for the molecular basis of HMS. Since introgressions vary in size and represent a very small proportion of the hybrid genome, they may or may not cover the factor responsible for the disruption and a range of fertility phenotypes results. For this reason, introgressions have been classically used in the mapping of HMS [16,30-32].

On the other hand, the mapping through introgressions may not detect small HMS sites when complex epistasis is present. Another limitation is that genetic rearrangements may show incompatibilities that do not really exist, for instance, when an event of transposition involving the introgression happens, we may lose an essential gene in one of the species and this can generate sterility or inviability that is not associated with hybrid incompatibilities [33]. In our case the use of introgressions was facilitated by the fact that previous work had already established the parental lines. In addition, QTL mapping had been previously done in the same region [16].

\section{HMS expression depends on genomic background}

In previous work, two of the HMS factors located on chromosome 3 were fine-mapped and characterized at the molecular level $[16,34,35]$. These factors have a large effect on HMS, but several other factors of small effect may be acting additively [36] or epistatically [15,37] in generating the hybrid incompatibility. These results support the view that that HMS is often a polygenic trait.

The polygenic nature of the hybrid sterility also accounts for the incomplete penetrance and variable phenotypic expression in each background. We tested three different backgrounds besides the stock simB used for the mapping. We find the same qualitative result, a reduction in fertility due to the presence of two $D$. mauritiana alleles of factor 1. However, the magnitude of this difference varies according to the background. In the background of line $w$; $e$, the presence of factor 1 when homozygous causes fertility to drop 30 fold, whereas in the w501 background fertility drops only 
twofold (Figure 4). This contrast indicates that there may be a complex network of negative epistatic interactions causing HMS. Thus, the effect of hybridization may depend on several factors that frequently vary among different lines of each species.

\section{Regulatory effects of HMS}

Information on gene expression is provided by the FlyAtlas [26] and refers to expression in different tissues of D. melanogaster. All genes contained in our interval are available in the dataset. Gene CG2358 (Spase 18-21) shows enrichment in salivary glands, male accessory glands, and larval salivary gland. Expression of this gene is up regulated in these tissues. Among the other three genes, agt shows a twofold enrichment in male accessory glands.

Recent studies of gene expression in hybrids have found that the misexpression of genes involved in spermatogenesis may cause sterility in hybrids $[38,39]$. Most of these genes are underexpressed in the hybrids relative to the parental species $[20,40]$, and this finding might reflect a disruption of gene interactions that are particular to each species. These results come from work where whole-genome hybrids were compared to both parental species, which is a situation different than our study. Here we used lines that bear a hybrid region considerably smaller (3R: 1,468,434..7,938,322), in the background of $D$. simulans (more specifically line $\operatorname{sim} B$ ). Most importantly, the segment that differs between the fertile and non-fertile lines is only $\sim 1.4 \mathrm{Mb}$ and contains only 174 known protein coding genes. Yet we find results qualitatively similar: the average number of down regulated genes in hybrids was more than double the average number of up regulated genes and both values were much greater than expected (Figure 5). Importantly, genes belonging to the Gene Ontology category of spermatogenesis are preferentially affected, with 30 targets showing differential expression.

Artieri et al. [41] showed that underexpressed genes in hybrids appear to evolve more rapidly than genes expressed normally in hybrids. This fact contributes to the idea that rapid evolution reduces gene similarity and potentially causes genetic incompatibilities.

\section{Molecular evolution of HMS}

The fine mapping described here defined a region as small as $9 \mathrm{~kb}$ that includes the candidate causing the great reduction in fertility in hybrid males of $D$. simulans and D. mauritiana. None of the genes present in this region are clearly involved in spermatogenesis, although signals of rapid evolution are present and help to suggest a candidate for factor 1. Gene agt is a small gene (576 bp) with high polymorphism within species and a number of nucleotide substitutions between species (Table 1). Among the fixed substitutions, four are synonymous and one is non-synonymous. Moreover, the region where agt is present shows very low conservation.

Similarly to agt, the gene Ovd (GA19777), recently described by Phadnis and Orr [9], lacked strong evidence of non-neutral evolution but proved to be the best candidate for the hybrid incompatibilities between the subspecies D. pseudobscura pseudobscura and D. pseudobscura bogotana. The authors found that this gene is involved in causing both segregation distortion in the $F_{1}$ and hybrid male sterility.

Identifying the normal function of a candidate gene within the parental species is of great interest when investigating the basis of hybrid incompatibilities. So far, no particular function can be attributed to genes involved in speciation. Some are enzymes, some are transcription factors, and others are structural proteins [42]. One possible explanation for the involvement of these varied classes of genes in reproductive isolation is that genetic substitutions accumulate over time, ultimately leading to enough divergence to cause genetic incompatibilities $[7,21]$. Nevertheless, the most common characteristics of genes involved in hybrid male sterility are signals of rapid evolution and positive selection within species [12,42].

One candidate for factor 1 in our study, gene agt, is reported as being involved in the repair and attenuation of the toxic and mutagenic effects of certain alkylating agents. Kooistra et al. [43] showed that the expression of agt suppresses transition mutations (G:C to A:T and vice-versa) in vivo. At the molecular level, agt is involved in methyltransferase activity. Apparently, this function does not have any clear association with reproduction for its disruption to lead to male sterility. Nevertheless, the gene may have as yet unidentified functions, or its enzymatic function may be deployed in some manner essential to hybrid male fertility.

In the mouse, the recently identified speciation gene $\operatorname{Prdm} 9$ is known to encode a meiotic histone H3 methyltransferase [10,44]. In the parental species, $\operatorname{Prdm} 9$ activates genes essential for meiosis and thus is essential for reproduction. The disruption of this function in hybrids of Mus m. musculus and Mus m. domesticus leads to male sterility, similarly to the phenotype of the $\operatorname{Prdm} 9^{-/-}$mutants. Similarly to $\operatorname{Prdm} 9$ and $O v d$, the earlier identified HMS gene $(\mathrm{OdsH})$ was recently reported to also encode a protein with putative DNA binding domain [45]. Thus, proteins that bind to chromatin and have possible regulatory roles may represent the most common class of factors whose disruption leads to hybrid incompatibilities. The gene Taf1 also functions as sequence-specific DNA binding protein and shows transcription factor activity. The fact that Taf1's 
function corresponds to what is described for most of the genes involved in hybrid male sterility may, per se, suggest this as a plausible candidate gene for factor 1 .

Across the Drosophila phylogeny, agt has undergone substitutions more often than other functional genes in the candidate region we mapped. Strikingly, substitutions in position 361, cited in the previous section, occurred in every clade since the split of D. melanogaster, and all lead to amino acid substitution. This information may indicate that agt is evolving rapidly and systematically changing with every branching event. Substitutions in position 361 are fixed within species and may be the key difference leading to the drop in fertility seen in hybrid males. Another observation is that agt shows unambiguous sorting of the three species of the simulans clade (Figure 3), as also observed for $\mathrm{OdsH}$, another gene involved in hybrid incompatibility between $D$. simulans and D. mauritiana [25]. Ongoing experiments focus on confirming the role of genes Taf1 or agt in causing the HMS via germ-line transformation rescue.

\section{Conclusions}

Our results suggest two candidate genes possibly leading to HMS between $D$. simulans and $D$. mauritiana. The mapping of such a complex phenotype down to a $9 \mathrm{~kb}$ region and to identifying candidate genes is an important achievement for the field and contributes to the knowledge of what classes of genes may cause HMS when disrupted in hybrids. Further experiments will investigate the functional role of Taf1 and agt in causing the decrease in fertility.

\section{Methods}

\section{Drosophila stocks}

D. simulans: (1) simB: $w$; nt; III (white; net; third chromosome homozygous and isogenic to that of line $13 \mathrm{w} 1$ $\times 1 \mathrm{JJ})$. The construction of $13 \mathrm{w} 1 \times 1 \mathrm{JJ}$ and $\operatorname{simB}$ was described earlier [34,46]; (2) sim $w$; e (white; ebony). All the stocks were provided by J. Coyne and maintained in the laboratory for several generations.

D. mauritiana: $w$ (white); $P\left[w^{+}\right]$, lines with independent $P$-element insertions on the third chromosome [47]. The $P\left[w^{+}\right]$inserts are semi-dominant markers with position effect, i.e., the wild form of white carried in the $P$-element produces an eye color between yellow and red, depending on the location of the $P$-insert.

The choice of lines to use for the mapping of factor 1 was based on the work of Tao et al. [16,34]. In that study hybrid lines between $D$. simulans and $D$. mauritiana were constructed. After several generations of backcrossing to the simB line (described above) and selecting for the colored-eye progeny, a piece of $D$. mauritiana's $3^{\text {rd }}$ chromosome of varying size was introgressed into the genome of $D$. simulans. A total of 231 introgression lines were created from a set of $28 \mathrm{D}$. mauritiana lines bearing one copy of $P\left[w^{+}\right]$independently inserted in the $3^{\text {rd }}$ chromosome. Details of the introgression scheme are in Tao et al. [[34], Figure 4], as well as the names given for the introgression lines.

Three lines composed of a simB background and one $P\left[w^{+}\right]$-tagged $D$. mauritiana introgression on the third chromosome were used for the mapping. The creation of these lines is described elsewhere [34,47].

\section{Genetic mapping}

Lines P32.8 (yellow eye) and P33.3 (red eye) were chosen for having $P$-element inserts flanking factor 1 $[47,48]$. Two generations were necessary to construct a heterozygote line with both $P$-inserts in cis: P32.8 females and $P 33.3$ males generated a proportion of offspring with both $P$-inserts in trans, which could be distinguished from the others by their dark-red eyes. Females with inserts in trans were crossed to simB males, and the darker-eye offspring selected again as bearing P32 and P33 inserts in cis (Figure 1). The $2 P$ construct carries a $D$. mauritiana introgression that covers the region where factor 1 had previously been located [31]. Other two factors identified as possible HMS (\#9 and \#10 - [16]) were previously located in regions covered by the $2 P$ construct we generated. However, the existence of these factors is not a source of influence on our results, as factor $\# 9$ is always present in every P32 recombinant line used here and factor \#10 is never included in the fine mapping (i.e. when we focused on recombinant lines bearing small introgressions). Thus, only the presence or absence of factor 1 may be associated to fertility or sterility.

The cross of $2 P$ females to simB males generates single- $P$ recombinants, which can be recognized by an eye color that is lighter than in the original $P$ lines; these carry D. mauritiana pieces of different sizes (Figure 1). The ideal $2 P$ design uses recombinant lines having either of the $P$-inserts from the parental lines, thus flanking factor 1 from both sides. However, in the present case, a reliable separation of $P 33$ recombinants from $2 P$ non-recombinants based on eye color was not possible. We thus decided to establish lines only from P32 recombinants.

Each recombinant male was crossed to five females of $\operatorname{sim} B$ in order to establish recombinant lines bearing heterozygous D. mauritiana introgressions. Because a single copy of the introgression does not harm male fertility, these lines were maintained through males $\times \operatorname{sim} B$ females in every generation. Moreover, since Drosophila males do not have recombination, the transmission through males assures the integrity of introgressions, and hence the perpetuation of the recombinant line. 
Males from stable recombinant lines were then taken for genotyping (assessment of introgression length) and fertility tests.

\section{Fertility assay}

Although the lack of recombination in Drosophila males can be very convenient for designing genetic experiments, it allows spontaneous mutations to accumulate through Muller's ratchet. Some of these mutations may cause sterility when in homozygosity. The frequency of spontaneous sterility was estimated as $\sim 1.5 \%[34,49]$ which is capable of blurring the fertility tests. In order to circumvent this concern and bring factor 1 to a homozygote state, we generated trans-heterozygote males from two independently raised $P\left[\mathrm{w}^{+}\right]$stocks, i.e., males from $P 32$ recombinant lines were crossed to females from P45.6 (named the tester stock), and the male offspring with this combination of $P$-inserts (darkred eyes) were selected for fertility tests (Figure 1). In this way, no spontaneous mutation occurring in the original P32.8 or P45.6 will be homozygous, whereas factor 1 may or may not be homozygous depending on the size of the introgression in each case.

Ten trans-heterozygote males were selected from each cross for the fertility tests. The typical fertility analysis used in previous work is based on the number of motile sperm present in seminal vesicles [50]. Here we follow the assay by Tao et al. [31,34], which is based on counting viable offspring derived from trans-heterozygote males. This is a more quantitative method that allows us to separate by sex (in order to investigate the occurrence of sex-ratio distortion) and eye color. Each of 10 trans-heterozygote males was crossed to three virgin females of $D$. simulans $w$; $e$ for seven days. After this period, females were discarded and males were collected for single-fly genotyping.

Offspring were counted up to the $20^{\text {th }}$ day and males classified as fertile or quasi-sterile. We observed that two copies of factor 1 cause either a severe drop in fertility or complete sterility. Recombinant lines were classified as quasi-sterile when their trans-heterozygote males had on average zero to 30 offspring. This range was empirically chosen, as outside this range the fertility jumps to an average of 120 offspring or more. A negligible number of males had progeny numbers between these two categories and were removed from the analysis.

\section{Single-fly genotyping}

After the seven-day mating period with $w$; $e$ females, trans-heterozygote males were collected and placed one in each well of 96-well plates. Grinding solution was added in each well $(40 \mu \mathrm{l}$ of $10 \mathrm{mM}$ Tris $\mathrm{pH} 8.2,1 \mathrm{mM}$ EDTA, $25 \mathrm{mM} \mathrm{NaCl}, 0.2 \mathrm{mg} / \mathrm{ml}$ Proteinase K) and flies were homogenized. The plates were incubated in $65^{\circ}$ for $30 \mathrm{~min}, 95^{\circ}$ for $2 \mathrm{~min}$ and chilled on ice briefly before being stored at $-20^{\circ}$.

The genotyping made use of molecular markers from various sources. First, allele-specific oligonucleotide markers previously developed (ASO [51]) were used as external markers in order to delimitate the region. We then designed additional ASO markers as the genetic dissection of the HMS region progressed. The ASO probes are pairs of 15 -mers that recognize the same sequence, but carry one or more SNPs (single nucleotide polymorphisms) between $D$. simulans and $D$. mauritiana. The steps for designing ASO probes are described in detail by Tao et al. [34]. In their work, primers were designed using the genome of $D$. melanogaster as template. However, in the present work we could take advantage of the genome project completed for D. simulans, as well as some regions of $D$. mauritiana obtained from 454 Life Sciences sequencing carried out at the Genome Center at Washington University in St. Louis.

Other markers were based on PCR success/failure using species-specific primers and PCR products with species-specific sizes. In the first case, triads of primers were designed in order to have one of them, either forward or reverse, annealing perfectly to both species, and a pair showing species-specific annealing. Additional file 6 lists the molecular markers used, as well as the primers, probes, and experimental conditions for their use. All oligonucleotides were designed using the online tool of Primer 3 http://frodo.wi.mit.edu.

During the final mapping step, we sequenced $20 \mathrm{~kb}$ spanning the region bearing factor 1 for $\operatorname{sim} B$, mau12, and $w ; e$. The $20 \mathrm{~kb}$ region was split into seven $\sim 3 \mathrm{~kb}-$ pieces in order to facilitate PCR reaction and downstream methods. We extracted DNA from $\sim 10$ flies of each stock using DNeasy (QIAgen). PCR reaction was performed using TaKaRa LA Taq (Takara Bio Inc.) and the protocol: $94^{\circ}$ for 1 minute; 30 cycles of $94^{\circ}$ for 15 seconds, $55^{\circ}$ for 30 seconds, $68^{\circ}$ for 5 minutes and extension in $72^{\circ}$ for 10 minutes. PCR products were cleaned with ExoSAP-it (USB). In total, 36 pairs of primers were used to sequence the seven pieces. This coverage provided a complete set of SNPs and indels and served as a reliable and straightforward source for genotyping.

\section{Molecular characterization of the candidate interval}

We sequenced the $20 \mathrm{~kb}$ extent of the candidate region for the lines $\operatorname{sim} B, w ; e$, and mau12. For a length of $1 \mathrm{~kb}$ encompassing the coding and flanking regions of gene agt (CG1303), an additional 15 strains of D. simulans from different locations across Africa and the Americas, and 17 strains of D. mauritiana collected in 2006 (kindly provided by Dr. Maria Margarita Ramos), were 
sequenced. Regions of $2.2 \mathrm{~kb}$ (Taf1), $3 \mathrm{~kb}$ (CG1307) and $2.4 \mathrm{~kb}$ (CG2358) were sequenced for a subset of 8 strains of D. simulans and 8 of D. mauritiana. Contig assembly was performed with Sequencher 3.0 (Gene Codes Corporation, Ann Arbor, MI, USA) and alignment was performed using ClustalW software [52].

Molecular genetic analyses, including the McDonaldKreitman test, were performed with DnaSP [53]. Phylogenetic tree reconstruction was performed for each gene's coding region separately, using maximum likelihood with phyML software [54], after running jModelTest [55] to determine the best fitting model to each alignment. The phylogeny obtained for each gene was used in the detection of positive selection with the software PAML [56].

\section{Gene expression analysis}

Genome-wide microarray analyses of gene expression of fertile and non-fertile lines were performed. The lines used in this essay were recombinant lines generated from an early step of the mapping process, when introgressions were covering a large region of chromosome $3 R$. Five recombinant lines were chosen and crossed to the tester stock P45.6 in order to bring factor 1 to a homozygous condition. Lines \#96 and \#102 were completely sterile, but were genotyped as having introgression of same size as quasi-sterile lines \#143 and \#188. For this reason, these lines were merged in the same group, non-fertile, and compared to the only normally fertile line \#225 (Figure 6A). Line \#225 bears a smaller introgression generated by recombination in the mother. The breakpoint excluding factor 1 from this line was located in between markers Antp and CG31195, but not precisely determined at this early step of the mapping. Thus, the introgressions present in these lines are identical at their 3' end but differ at the 5' end. Except for these differences in the amount of introgressed material and the consequential presence or absence of factor 1 and other elements within the introgressed region, these lines are genetically identical.

Microarrays were $\sim 18,000$-feature cDNA arrays spotted with $D$. melanogaster cDNA PCR products. Total RNA was extracted from whole flies using TRIzol (Life Technologies) and microarray analyses were performed with standard protocols previously described [57]. Using RNA from testis would focus the results on the specific effects of factor 1 on spermatogenesis, but on the other hand, would not give any information about the effects of factor 1 on genes that are exclusively expressed in other tissues. The microarray design implemented in this study is shown in Figure 6B.

The cDNA synthesis, the labelling with fluorescent dyes ( $\mathrm{Cy} 3$ and $\mathrm{Cy} 5)$, and the hybridization reactions were carried out using 3DNA protocols and reagents

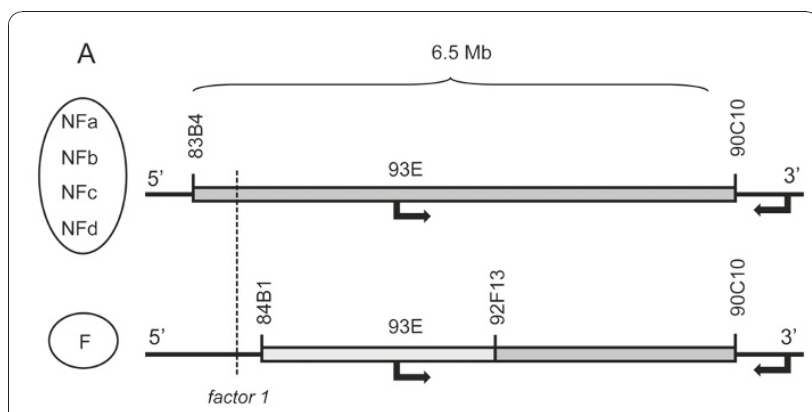

B

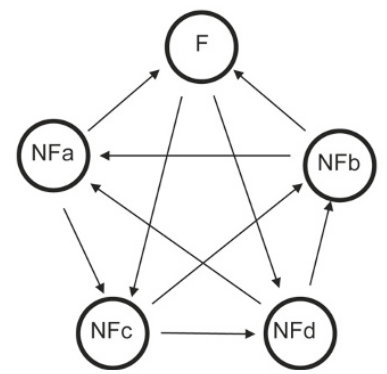

Figure 6 Microarray design comparing fertile and non-fertile lines. (A) Design for the microarray experiment comparing gene expression in one fertile and four non-fertile lines. The fertile line bears an introgression that may or may not include the light-gray region, but definitely excludes the portion where factor 1 is located (83B4-84B1). Black arrows indicate inversion previously known in D. simulans and D. mauritiana, in relation to D. melanogaster. (B) Microarray design showing all the comparisons among lines. In our design, four independently obtained lines with non-fertile phenotypes were compared with a fertile reference. These lines only differ in the presence of a small segment of $D$. mauritiana where factor 1 is present. In the fertile line this segment is present in heterozygosity with the homologous segment from $D$. simulans, whereas the segment is homozygous D. mauritiana/D. mauritiana in the four non-fertile lines.

(Genisphere). Slides were scanned using an Axon 4000B scanner (Axon Instruments) and GenePix Pro 6.0 software. Foreground Fluorescence of dye intensities was normalized by the Loess method in the R Limma library. Stringent quality-control criteria were used to ensure reliability of foreground intensity reads for both Cy5 and $\mathrm{Cy} 3$ channels. These conservative criteria were the following: ( $([$ F635Median - B635] $>4 *[$ B635 SD] OR [F532 Median - B532] $>4 *[$ B532 SD]) AND ([\% > B635 $+2 \mathrm{SD}]>70$ OR $[\%>\mathrm{B} 532+2 \mathrm{SD}]>70)$ AND $([\mathrm{F} 635 \%$ Sat.] < 45 AND [F532 \% Sat.] < 45) AND ([B532 Median] $<4 *$ [B635 Median] AND [B635 Median] $<4^{*}[$ B532 Median]) AND ([Sum of Medians (635/532)] > 100) AND ([SNR 635] $>2$ AND [SNR 532] $>2$ ) AND ([Rgn R2 $(635 / 532)]>0.5)$ AND ([Circularity] > 0.45)), where F532 and F635 denote the foreground fluorescence intensities, B532 and B635 denote the background fluorescence intensities, SNR 532 and SNR 635 denote signal to noise ratio for $\mathrm{Cy} 3$ and $\mathrm{Cy} 5$, respectively. Rgn $\mathrm{R} 2$ and circularity denote the spot specific coefficient of 
determination and spot specific circularity as calculated by the GenePix software.

The significance of variation in gene expression due to the introgressed segment causing HMS was assessed with linear models in Limma and with the Bayesian Analysis of Gene Expression Levels (BAGEL). FDRs were estimated based on the variation observed when randomized versions of the original dataset were analyzed.

The data discussed in this publication have been deposited in NCBI's Gene Expression Omnibus [58] and are accessible through GEO Series accession number GSE25339 http://www.ncbi.nlm.nih.gov/geo/query/acc. cgi?acc=GSE25339.

\section{Additional material}

\section{Additional file 1: Recombinant lines and phenotypes at the final} step of mapping factor $\mathbf{1}$. Detailed localization of factor 1 according to four recombinant males showing introgression of similar sizes and different phenotypes. Only one chromosome is shown for each male. Recombinant break points were identified based on SNPs within genes (large font) or in the intergenic region (small font). Phenotype is given by the mean progeny size and standard error below each chromosome. The mean is based on 10 homozygous males from each recombinant line (see Methods). Finally, we show the position of factor 1 according to the annotated $D$. simulans genome.

Additional file 2: Graphic scheme of the region where factor 1 is located. (A) Graphic scheme showing the $9 \mathrm{~kb}$ mapped region and the genes found within it (gene span and mRNA). Note that only seven of Taf1's 16 exons are contained in the region. The arrows show approximate location and relative size of indels found in the upstream region of gene agt. (B) alignment of a portion of the upstream region of gene agt for different populations of D. simulans and D. mauritiana.

Additional file 3: Inter-species conservation across the mapped region. Graph from UCSC alignments showing the degree of conservation across species in D. melanogaster group and close species. The reference sequence represents the species $D$. melanogaster. A range of $20 \mathrm{~kb}$ is shown. Coding regions show much higher conservation than introns and intergenic regions. However, the coding region of gene agt shows low conservation across species (yellow stripe).

Additional file 4: List of genes showing misexpression in at least one of the non-fertile lines

Additional file 5: List of genes with misexpression congruent in all four non-fertile lines. Negative values mean that genes were down regulated in the non-fertile lines in relation to the fertile one and positive values mean up regulation in the non-fertile lines. The 5 down regulated genes contained within the introgressed segment are shown in red.

Additional file 6: List of molecular markers used for mapping factor 1

\section{Acknowledgements}

We thank Yun Tao for sharing the intellectual background of this work. Our progress was greatly enhanced by his always-generous contributions with ideas, material and deep knowledge of the subject; also David Miller and Thomas Kaufman for providing the piggyBac vector we have been using for the transformations. Nathan Eckstrand and Kalsang Namgyal helped with technical assistance. We would like to thank three anonymous reviewers for suggestions that greatly improved the manuscript. This work was supported by NIH grant GM065169.

\section{Authors' contributions}

LOA carried out the design of the study, genetic crosses, analysis of phenotype and genotype, and drafted the manuscript. HM performed the experiment in different genetic backgrounds, helped with analyses, and gave suggestions on the draft. BL designed, performed and analyzed the microarray experiment, and helped to draft the manuscript. DLH participated in the design and coordination of the study, and gave suggestions on the

draft. All authors read and approved the final manuscript.

Received: 26 July 2010 Accepted: 14 December 2010

Published: 14 December 2010

\section{References}

1. Dobzhansky T: Speciation as a stage in evolutionary divergence. Am Nat 1940, 74:312-321.

2. Muller HJ: Bearing on the Drosophila work on systematics. In The New Systematics. Edited by: Huxley JS. Clarenton Press, Oxford; 1940.

3. Ting C-T, Tsaur S-C, Wu M-L, Wu C-l: A rapidly evolving homeobox at the site of a hybrid sterility gene. Science 1998, 282:1501-1504.

4. Wu C-I, Ting C-T: Genes and speciation. Nature Reviews Genetics 2004 5:114-122

5. Presgraves DC, Balagopalan L, Abmayr SM, Orr AH: Adaptive evolution drives divergence of a hybrid inviability gene between two species of Drosophila. Nature 2003, 423:715-719.

6. Tang S, Presgraves DC: Evolution of the Drosophila nuclear pore complex results in multiple hybrid incompatibilities. Science 2009, 323:779-782.

7. Barbash DA, Awadalla P, Tarone AM: Functional divergence caused by ancient positive selection of a Drosophila hybrid incompatibility locus. PLoS Biology 2004, 2:839-848.

8. Sawamura K, Yamamoto M-T: Cytogenetical localization of Zygotic hybrid rescue (Zhr), a Drosophila melanogaster gene that rescues interspecific hybrids from embryonic lethality. Mol Gen Genet 1993, 239:441-449.

9. Phadnis N, Orr HA: A single gene causes both male sterility and segregation distortion in Drosophila hybrids. Science 2009, 323:376-379.

10. Mihola O, Trachtulec Z, Vlcek C, Schimenti JC, Forejt J: A mouse speciation gene encodes a meiotic histone $\mathrm{H} 3$ methyltransferase. Science 2009, 323:373-375.

11. Wittbrodt J, Adam D, Malitschek B, Maueler W, Raulf F, et al: Novel putative receptor tyrosine kinase encoded by the melanoma-inducing Tu locus in Xiphophorus. Nature 1989, 341:415-421.

12. Coyne JA, Orr HA: Speciation. Sunderland, MA: Sinauer Associates; 2004

13. Mallet J: Hybridization as an invasion of the genome. TREE 2005, 20:229-237.

14. Perez DP, Wu C-I: Further characterization of the hybrid sterility gene, Odysseus (Ods), in the Drosophila simulans clade: one gene is not enough. Genetics 1995, 140:201-206.

15. Orr HA, Irving S: Complex epistasis and the genetic basis of hybrid sterility in the Drosophila pseudoobscura Bogota-USA hybridization. Genetics 2001, 158:1090-1100

16. Tao Y, Zeng Z-B, Li J, Hartl DL, Laurie CC: Genetic dissection of hybrid incompatibilities between Drosophila simulans and D. mauritiana. II. Mapping hybrid sterility loci on the third chromosome. Genetics 2003 164:1399-1418.

17. Orr AH: The population genetics of speciation: the evolution of hybrid incompatibilities. Genetics 1995, 139:1805-1813.

18. Mallet J: What does Drosophila genetics tell us about speciation? TREE 2006, 21:386-393.

19. Orr AH: Haldane's rule. Annu Rev Ecol Syst 1997, 28:195-218.

20. Haerty W, Singh RS: Gene regulation divergence is a major contributor to the evolution of Dobzhansky-Muller incompatibilities between species of Drosophila. Mol Biol Evol 2006, 23:1707-1714.

21. Presgraves DC: The molecular evolutionary basis of species formation. Nature Rev Gen 2010, 11:175-180.

22. Wassarman DA, Aoyagi N, Pile LA, Schlag EM: TAF250 is required for multiple developmental events in Drosophila. Proc Natl Acad Sci USA 2000, 97:1154-1159.

23. Palopoli MF, Davis AW, Wu C-I: Discord between the phylogenies inferred from molecular vs. functional data: uneven rates of functional evolution or low levels of gene flow? Genetics 1996, 144:1321-1328. 
24. Wang RL, Wakeley J, Hey J: Gene flow and natural selection in the origin of Drosophila pseudoobscura and close relatives. Genetics 1997, 147:1091-1106.

25. Ting C-T, Tsaur SC, Wu Cl: The phylogeny of closely related species as revealed by the genealogy of a speciation gene, Odysseus. Proc Natl Acad Sci USA 2000, 97:5313-5316.

26. Chintapalli VR, Wang J, Dow JAT: Using FlyAtlas to identify better Drosophila models of human disease. Nature Genetics 2007, 39:715-720

27. Kliman RM, Andolfatto P, Coyne JA, Depaulis F, Kreitman M, et al: The population genetics of the origin and divergence of the Drosophila simulans complex species. Genetics 2000, 156:1913-1931.

28. Mayr E: Systematics and the Origin of Species. Columbia University Press, New York; 1942

29. Ballard JW: When one is not enough: Introgression of mitochondrial DNA in Drosophila. Mol Biol Evol 2000, 17:1126-1130.

30. Wu C-I, Hollocher H: Subtle is nature: the genetics of differentiation and speciation. In Endless Forms: Species and Speciation. Edited by: Berlocher S. Oxford Univ. Press, Oxford: 1998:339-351.

31. Tao Y, Hartl DL, Laurie CC: Sex-ratio segregation distortion associated with reproductive isolation in Drosophila. Proc Natl Acad Sci USA 2001, 98:13183-13188

32. Masly JP, Presgraves DC: High-Resolution Genome Wide Dissection of the Two Rules of Speciation in Drosophila. PLoS Biol 2007, 5:e243.

33. Masly JP, Jones CD, Noor MAF, Orr HA: Gene transposition as a cause of hybrid sterility. Science 2006, 313:1448-1450.

34. Tao Y, Chen S, Hartl DL, Laurie CC: Genetic dissection of hybrid incompatibilities between Drosophila simulans and D. mauritiana. I. Differential accumulation of hybrid male sterility effects on the $X$ and autosomes. Genetics 2003, 164:1383-1397.

35. Tao Y, Masly JP, Araripe L, Ke Y, Hartl DL: A sex-ratio system in Drosophila simulans. I: An autosomal suppressor. PLOS Biol 2007, 5:e292.

36. Naveira HF, Maside XR: The genetics of hybrid male sterility in Drosophila. In Endless Forms: Species and Speciation. Edited by: Howard DJ, Berlocher SH. Oxford University Press, New York; 1998:330-338.

37. Sawamura K, Roote J, Wu C-I, Yamamoto M-T: Genetic complexity underlying hybrid male sterility in Drosophila. Genetics 2004, 166:789-796.

38. Michalak P, Noor MAF: Genome-wide patterns of expression in Drosophila pure-species and hybrid males. Mol Biol Evol 2003, 20:1070-1076.

39. Catron DJ, Noor MAF: Gene expression disruptions of organism versus organ in Drosophila species hybrids. PLOS ONE 2008, 3:e309.

40. Moehring AJ, Teeter KC, Noor MAF: Genome-Wide Patterns of Expression in Drosophila Pure Species and Hybrid Males. II. Examination of MultipleSpecies Hybridizations, Platforms, and Life Cycle Stages. Mol Biol Evol 2007, 24(1):137-145.

41. Artieri CG, Haerty W, Singh RS: Association between levels of coding sequence divergence and gene misregulation in Drosophila male hybrids. J Mol Evol 2007, 65:697-704.

42. Orr AH, Masly JP, Phadnis N: Speciation in Drosophila: from phenotypes to molecules. J Heredity 2006, 98:103-110.

43. Kooistra R, Zonneveld JB, Watson AJ, Margison GP, Lohman PH, Pastink A Identification and characterisation of the Drosophila melanogaster 0 6alkylguanine-DNA alkyltransferase cDNA. Nucleic Acids Res 1999, 27:1795-1801.

44. Mihola O, Forejt J, Trachtulec Z: Conserved alternative and antisense transcripts at the programmed cell death 2 locus. BMC Genomics 2007, 8:20.

45. Bayes JJ, Malik HS: Altered heterochromatin binding by a hybrid sterility protein in Drosophila sibling species. Science 2009, 326:1538-1541.

46. Liu J, Mercer JM, Stam LF, Gibson GC, Zeng ZB, Laurie CC: Genetic analysis of a morphological shape difference in the male genitalia of Drosophila simulans and D. mauritiana. Genetics 1996, 142:1129-1145.

47. True JR, Mercer JM, Laurie CC: Differences in crossover frequency and distribution among three sibling species of Drosophila. Genetics 1996, 142:507-523.

48. Araripe $L O$, Eckstrand ND, Hartl DL, Tao Y: Flanking regions of $P$-elements inserted in the $3^{\text {rd }}$ chromosome of Drosophila mauritiana. Dros Inf Ser 2006, 89:54.

49. True JR, Weir BS, Laurie CC: A genome-wide survey of hybrid incompatibility factors by the introgression of marked segments of Drosophila mauritiana chromosomes into D. simulans. Genetics 1996 142:819-837
50. Davis AW, Wu C-I: The broom of the sorcerer's apprentice: the fine structure of a chromosomal region causing reproductive isolation between two sibling species of Drosophila. Genetics 1996, 143:1287-1298.

51. Saiki RK, Bugawan TL, Horn GT, Mullis KB, Erlich HA: Analysis of enzymatically amplified-globin and HLA-DQ DNA with allele-specific oligonucleotide probes. Nature 1986, 324:163-166

52. Thompson JD, Higgins DG, Gibson TJ: CLUSTAL W: improving the sensitivity of progressive multiple sequence alignment through sequence weighting, position-specific gap penalties and weight matrix choice. Nucleic Acids Res 1994, 22:4673-4680.

53. Librado P, Rozas J: DnaSP v5: A software for comprehensive analysis of DNA polymorphism data. Bioinformatics 2009, 25:1451-1452.

54. Guindon S, Pascual O: A simple, fast, and accurate algorithm to estimate large phylogenies by maximum likelihood. Syst Biol 2003, 52:696-704.

55. Posada D: jModelTest: Phylogenetic model averaging. Mol Biol Evol 2008, 25:1253-1256.

56. Yang Z: Phylogenetic analysis by maximum likelihood (PAML), Version 3.0. University College London, London, England; 2000.

57. Lemos B, Araripe LO, Hartl DL: Polymorphic Y chromosomes harbor cryptic variation with manifold functional consequences. Science 2008, 319:91-93.

58. Edgar R, Domrachev M, Lash AE: Gene Expression Omnibus: NCBI gene expression and hybridization array data repository. Nucleic Acids Res 2002, 30(1):207-210

doi:10.1186/1471-2148-10-385

Cite this article as: Araripe et al:: Fine-scale genetic mapping of a hybrid sterility factor between Drosophila simulans and D. mauritiana: the varied and elusive functions of "speciation genes". BMC Evolutionary Biology 2010 10:385.

\section{Submit your next manuscript to BioMed Central and take full advantage of:}

- Convenient online submission

- Thorough peer review

- No space constraints or color figure charges

- Immediate publication on acceptance

- Inclusion in PubMed, CAS, Scopus and Google Scholar

- Research which is freely available for redistribution 